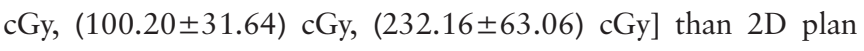
$(\mathrm{P}<0.05)$.

Conclusions Image-guidance brachytherapy of cervical cancer is helpful to detect hidden uterine perforation. When uterine perforation occurs, 3D plan can basically meet the clinical needs and is significantly better than $2 \mathrm{D}$ plan.

\section{EPV072/\#413 SUTURE GRANULOMA MIMICKING STAGE IB1 CERVICAL CANCER RECURRENCE ON VESICAL- UTERINE SPACE AFTER RADICAL TRACHELECTOMY}

${ }^{1} \mathrm{PE}$ Carvalho De Cillo*, ${ }^{1} \mathrm{H}$ Arisawa, ${ }^{2} \mathrm{O}$ Rangel, ${ }^{1} \mathrm{MGB}$ Uyeda. ${ }^{1}$ Universidade Federal de São Paulo, Gynecolgic Oncology, São Paulo - SP, Brazil; ${ }^{2}$ Universidade Federal de São Paulo, Gynecologic Oncology, São Paulo, Brazil

\subsection{6/ijgc-2021-IGCS. 140}

Objectives Introduction: Suture granuloma is a rare benign tumor caused by suture material, which usually appears several months or years after surgery. It may look identical to tumor relapse or metastatic disease on CT, PET scans and MRI, requiring histopathologic confirmation. An electronic literature search was undertaken using Medline, PubMed and Cochrane library databases for cerclage suture granuloma cases, using terms 'cerclage', 'suture granuloma', 'cervical cancer' 'metastasis' and boolean operators AND or OR, without any findings. Methods Presentation of case: 31 years-old with cervical cancer IB1 in 2014. She underwent radical trachelectomy, laparoscopic limphadenectomy and cerclage as fertility-sparing treatment. The patient attended for regular follow-up during 4 years, without signs of recurrence. 6 years after treatment, presented vaginal bleeding and fetid discharge. Physical examination: $1 \mathrm{~cm}$ exophytic bleeding lesion on anterior cervical-vaginal recess. Two biopsies were realized without any concluding findings. Pelvic MRI evidenced a cystic lobulated formation with thick walls, vascularized septs presenting wide contact with vesical trigon measuring $2.2 \times 1.5 \times 2.4 \mathrm{~cm}$, and an enlarged lymphnode in internal iliac chain of $1.3 \mathrm{~cm}$. Considering the risk of malignancy, the patient was submitted to laparoscopic resection of the lesion and lymphnode dissection.

Results The final analysis resulted in granulation tissue with no signs of malignancy in lesion or in lymphnode, thus excluding the hypothesis of malignant recurrence or metastasis.

Conclusions Discussion: Foreign body granuloma may look identical to tumor relapse or metastatic disease on image exams, thus requiring histopathological confirmation. However, the hypothesis of granulomatous reactions involving cerclage non-absorbable suture must be a differential diagnosis in clinical practice.

\section{EPV073/\#423 AN OVERVIEW OF CERVICAL CANCER EPIDEMIOLOGY AT THE ONCOLOGY GYNECOLOGY CENTER OF SANTA MARCELINA HOSPITAL- BRAZIL}

S Sanches*, M Mesquita, J Barbosa, T Almeida, M Brandão, M Silva, C Sousa, C Gomez, I Manchini. Casa de Saúde Santa Marcelina, Gynecologic Oncology, Sao Paulo, Brazil
Objectives Evaluate clinical results of patients with cervical cancer, based on statistics from the Oncology Gynecology Center of Santa Marcelina Hospital in Sao Paulo, Brazil, between 2012 to 2018 .

Methods Retrospective analysis of 370 patients' medical records, who were followed up. The data table included the following variables: age at diagnosis, symptoms, tobacco use, staging, treatments, fatal outcome, and histological type.

Results The median age of patients at diagnosis was 51 years. The major symptom was vaginal bleeding (53.65\%). Presence of smoking was reported by $33 \%$ of the patients. The histological distribution showed predominance of epidermoid carcinoma (83.6\%) over adenocarcinoma (13.8\%). About the diagnosis, $4.2 \%$ of the patients were in stage IA1, followed by IA2 2.2\%; IB1 $8.4 \%$; IB2 $6.5 \%$; IB3 1\%; IIA1 1.16\%; IIA2 2.7\%; IIB 15.4\%; IIIA 1.4\%; IIIB 41.9\%; IIIC1 1\%; IIIC2 0.84 ; IVA $5.4 \%$ and IVB $8.9 \%$. Only $20 \%$ of the patients underwent surgical treatment and the most frequent therapeutic option were radiotherapy and concomitant chemotherapy $(80 \%)$. The relapse rate was $23,24 \%$, mostly in the vagina, and about $40 \%$ of the patients had a fatal outcome in our review.

Conclusions Based on these data table, vaginal bleeding as the major symptom, the high rate of IIIB stage diagnosis, and of the fatal outcome, may be an indicative of late diagnosis of this population confirming reports from developing countries. As cervical cancer has a chance of cure if diagnosed at early stages, the results demonstrate the need for investments in educational initiatives to raise awareness among the public about the importance of cervical cancer screening.

\section{EPV074/\#427 CERVICAL CANCER: MULTICENTRIC EPIDEMIOLOGICAL STUDY}

${ }^{1} \mathrm{R}$ Arfaoui, ${ }^{2} \mathrm{MA}$ Ferjaoui ${ }^{*},{ }^{3} \mathrm{Y}$ Berrazega, ${ }^{4} \mathrm{~S}$ Khedhri, ${ }^{2} \mathrm{~K}$ Abdessamia, ${ }^{4} \mathrm{M}$ Malek, ${ }^{4} \mathrm{~K}$ Neji, ${ }^{2} \mathrm{H}$ Gmara. ${ }^{1}$ Tunis military hospital, Department of Gynecology and Obstetrics, Tunis, Tunisia; ${ }^{2}$ Tunis maternity center, Department B of Gynecologic Surgery, Tunis, Tunisia; ${ }^{3}$ Abderrahmen mami university hospital, Department Medical Oncology, Tunis, Tunisia;

${ }^{4}$ Tunis Maternity Center, Department B of Gynecologic Surgery, Tunis, Tunisia

\subsection{6/ijgc-2021-IGCS.142}

Objectives To determine the epidemiological profile of cervical cancer in Tunisia and to specify the cost of treating the disease in order to develop an effective prevention strategy.

Methods It is a retrospective descriptive, multicenter study conducted in 6 obstetrical gynecology departments over a four-year period from January 1, 2016 to December 31,2019.

Results The number of all-stage cervical cancer in the different centers was 655 cases over a four-year period; which is equivalent to 166 cases/year. The average age of our patients was 53.5 years. More than half of our population did not have health insurance, and 38\% were illiterate. The average age of sexual activity was 22.2 years. Cervical smear screening was performed in only 35 patients (17.9\% of cases). The average consultation time in the study population was 5.6 months. The most frequent reason for consultation was metrorrhagia in $63 \%$. A clear predominance of squamous cell carcinoma $(82 \%)$ was noted. Tumors were classified according to the FIGO 2009 classification: $23.5 \%$ were diagnosed at an early stage $(<\mathrm{IB} 1)$ and $76.3 \%$ at advanced stages (IB2 up to IV). 